\title{
Evaluation of Downstaging, Visual Inspection with Acetic Acid and Lugol's Iodine in Sareening of Cenvical Cancer
}

\author{
${ }^{1}$ Kamal Patil, ${ }^{2}$ Lakshita Lumb, ${ }^{1}$ MK Swamy, ${ }^{3}$ Geeta Durdi \\ ${ }^{1}$ Professor, Department of Obstetrics and Gynecology, Jawaharlal Nehru Medical College, Belgaum, Karnataka, India \\ ${ }^{2}$ Postgraduate Student, Department of Obstetrics and Gynecology, Jawaharlal Nehru Medical College, Belgaum, Karnataka, India \\ ${ }^{3}$ Associate Professor, Department of Obstetrics and Gynecology, Jawaharlal Nehru Medical College, Belgaum, Karnataka, India
}

Correspondence: Kamal Patil, Professor, Department of Obstetrics and Gynecology, Jawaharlal Nehru Medical College Belgaum-590010, Karnataka, India, Phone: 0831-2472510, Fax: 0831-2470759, e-mail: kamalpatil1967@yahoo.co.in

\section{ABSTRACT}

Objectives: To detect and compare the sensitivity and specificity of downstaging and visual inspection with acetic acid (VIA) and with Lugol's iodine (VILI).

Methods: Screening with downstaging, VIA and VILI were done on 680 women who fulfilled the inclusion criteria at a primary health center attached to the university hospital, over a period of one year. Those who had abnormal results in downstaging, VIA and VILI were biopsied and sensitivity, specificity, negative predictive value and positive predictive value were calculated.

Results: The sensitivities of downstaging, VIA and VILI were 54.55, 63.64 and $90.91 \%$ and specificities were 93.42, 95.07 and 97.46\% respectively. The positive predictive value of downstaging, VIA and VILI were $12.01,17.5$ and $35.7 \%$ and negative predictive values were 99.2, 99.37 and $98.85 \%$ respectively.

Conclusion: VIA and VILI are suitable primary screening procedures as compared to downstaging because of high sensitivity and specificity. The negative predictive value of downstaging, VIA and VILI is better than the positive predictive value. Downstaging can be used in rural areas where acetic acid and Lugol's iodine are not available.

Keywords: Cervical cancer screening, Downstaging, Visual inspection with acetic acid (VIA), Visual inspection with Lugol's iodine (VILI).

\section{INTRODUCTION}

Worldwide, approximately 5,00,000 new cases of cervical cancer (approximately one case per minute) are diagnosed, and 275,000 related deaths occur annually, making it the second or third most common female cancer and cancer related cause of mortality. ${ }^{1}$ It accounts for one-tenth of all cancer deaths in women. ${ }^{1}$ In India, an estimated 1.5 lakh women develop cervical cancer annually, which is about $16 \%$ of the world annual incidence. Thus, cervical cancer is an important public health problem that deserves urgent attention. ${ }^{2}$

Well-established screening programs for early detection of carcinoma cervix are present in developed countries, which is contrary to the scenario in developing countries like India where there is lack of infrastructure, trained health personnel and financial constraints. Keeping this in view, World Health Organization (WHO) has recommended screening tests for early detection of cervical cancer, namely downstaging, visual inspection with acetic acid (VIA), visual inspection with Lugol's iodine (VILI) and cytology (Pap smear).

Pap smear has been used as a method of screening for cervical cancer for several years. Review of literature has reported low sensitivity of 30 to $87 \%$ and specificity of 86 to $100 \%$ with cytology. ${ }^{3}$ Cytology based on screening models require technical capabilities and financial support that are not readily available in most developing countries. Hence, there is a need to implement low cost strategies like downstaging, visual inspection with acetic acid and visual inspection with Lugol's iodine.

VIA and VILI are simple, inexpensive screening tests that can be used for early detection of cervical precancerous lesions and early invasive cancer in low resource settings. The most important advantage is that it gives immediate result and no follow-up is required. However, VIA requires training by experts, freshly prepared acetic acid and white coherent source of light to conclude results, which is difficult in rural setup. In addition, it has high false-positive results and wide interobserver variations. VILI also requires comprehensive and competency based training of the health personnel. In comparison, downstaging only requires direct visualization of cervix by trained nurses and paramedical workers so that abnormal cases can be referred to higher centers for further evaluation.

This prospective study was taken up to detect carcinoma cervix, in early stages by downstaging, i.e. visual inspection of the cervix by trained nurses, and its comparison with VIA and VILI at the primary health center.

\section{MATERIALS AND METHODS}

This study was carried out at primary health center, Kinaye attached to KLES Dr Prabhakar Kore Hospital and Medical Reserch Center, Belgaum, Karnataka (a university hospital). 
It was conducted from 1st November 2008 to 31st October 2009. Around 680 married women between 25 and 65 years of age coming for health check-up at primary health center, Kinaye were enrolled in the study group, after obtaining written informed consent. Women with active vaginal bleeding, pregnancy and white discharge per vagina (infections) were excluded.

The procedure of downstaging, VIA and VILI was explained. The cervix was visualized for gross pathological features, under adequate light, by trained auxiliary nurse midwife (ANM). The results were concluded according to the WHO picture atlas. ${ }^{4}$ The cervix was cleaned with normal saline and freshly prepared 5\% acetic acid was taken on a swab stick and generously applied on the cervix. The cervix was inspected after one minute. Repeat application of 5\% acetic acid was done, if required. International Agency for Research on Cancer (IARC) guidelines were used for interpreting VIA test results. ${ }^{5}$ After results of VIA, the cervix was cleaned with normal saline and Lugol's iodine was generously applied on the cervix and was inspected under good light source. IARC guidelines were used for interpreting VILI test results. ${ }^{5}$ Biopsy was taken for downstaging, VIA and/or VILI positive women from the abnormal area using punch biopsy forceps. The specimen was sent to histopathology laboratory in formalin solution. Experienced pathologist analyzed the slides. Biopsy results were categorized as mild dysplasia (CIN 1), moderate dysplasia (CIN 2), severe dysplasia (CIN 3), and carcinoma in situ (CIN 3). In negative patients biopsy was taken in $1.6 \%$ of the cases.

The sensitivity and specificity of downstaging was compared with visual inspection with acetic acid (VIA) and visual inspection with Lugol's iodine (VILI) keeping cervical biopsy as a gold standard. Also, negative predictive value and positive predictive value were calculated for downstaging, VIA, VILI and cervical biopsy.

\section{RESULTS}

Around 680 women between 25 and 65 years of age who fulfilled the selection criteria were subjected to examination. Downstaging was positive in 50 out of 680 women (6.20\%) and this test detected nine cases with precancerous lesions, which included five CIN 1, three CIN 2 and one case of CIN 3 as shown in Table 1 . In the present study, 40 out of 680 women showed positive VIA results. VIA detected seven precancerous lesions, out of which three were CIN 1, two were CIN 2 and two were CIN 3 as shown in Table 2. Around 27 out of 680 women showed positive VILI results. VILI detected ten precancerous lesions, out of which five were CIN 1, three were CIN 2 and two were CIN 3 as shown in Table 3 . The sensitivities of downstaging, VIA and VILI were 54.55, 63.64 and 90.91\% respectively. The specificities of downstaging, VIA and VILI were 93.42, 95.07 and 97.46\% respectively. The positive predictive value (PPV) of downstaging, VIA and VILI were $12.01,17.5$ and $35.7 \%$ respectively. The negative predictive value (NPV) of downstaging, VIA and VILI were 99.2, 99.37 and $98.85 \%$ respectively. The sensitivity, specificity, positive and negative predictive value of downstaging, VIA and VILI are shown in Table 4.

Table 1 Correlation between downstaging and biopsy $(n=62)$

\begin{tabular}{|l|c|c|c|c|c|c|} 
Downstaging & \multicolumn{5}{|c|}{ Biopsy } & Total \\
\hline & Normal & $\begin{array}{c}\text { Cervicitis/ } \\
\text { Metaplasia }\end{array}$ & CIN 1 & CIN 2 & CIN 3 & \\
\cline { 2 - 7 } Positive & 7 & 34 & 5 & 3 & 1 & 50 \\
Negative & 4 & 7 & 0 & 0 & 1 & 12 \\
\hline Total & 11 & 41 & 5 & 3 & 2 & 62 \\
\hline
\end{tabular}

Table 2 Correlation between VIA and biopsy $(\mathrm{n}=62)$
\begin{tabular}{|l|c|c|c|c|c|c|} 
VIA & \multicolumn{5}{c|}{ Biopsy } & Total \\
& Normal & $\begin{array}{l}\text { Cervicitis/ } \\
\text { Metaplasia }\end{array}$ & CIN 1 & CIN 2 & CIN 3 & \\
\cline { 2 - 6 } & 8 & 25 & 3 & 2 & 2 & 40 \\
Positive & 4 & 14 & 3 & 1 & 0 & 22 \\
Negative & 12 & 39 & 6 & 4 & 0 & 62 \\
\hline Total & & & & &
\end{tabular}

Table 3 Correlation between VILI and biopsy $(n=62)$

\begin{tabular}{|l|c|c|c|c|c|c|} 
Pap smear & \multicolumn{5}{|c|}{ Biopsy } & Total \\
\hline & Normal & $\begin{array}{c}\text { Cervicitis/ } \\
\text { Metaplasia }\end{array}$ & CIN 1 & CIN 2 & CIN 3 & \\
\cline { 2 - 7 } Positive & 0 & 17 & 5 & 3 & 2 & 27 \\
Negative & 12 & 22 & 1 & 0 & 0 & 35 \\
\hline Total & 12 & 39 & 6 & 3 & 2 & 62 \\
\hline
\end{tabular}

Table 4

Diagnostic efficacy of tests

\begin{tabular}{|lcll|} 
& Downstaging & VIA & VILI \\
\hline Sensitivity & $54.55 \%$ & $63.64 \%$ & $90.91 \%$ \\
Specificity & $93.42 \%$ & $95.07 \%$ & $97.46 \%$ \\
PPV & $12 \%$ & $17.5 \%$ & $35.7 \%$ \\
NPV & $99.2 \%$ & $99.37 \%$ & $99.85 \%$ \\
\hline
\end{tabular}

\section{DISCUSSION}

Carcinoma cervix is the commonest genital cancer in women in developing countries. Well-established screening programs for early detection of carcinoma cervix are present in developed countries, which is not same in developing countries like India where there is lack of infrastructure, trained health personnel and financial constraints. It has become necessary to find alternative screening procedure to cytology, which has high sensitivity and specificity.

The present study was conducted at a primary health center and downstaging, VIA and VILI were performed in all women who fulfilled the inclusion criteria. Downstaging was positive in 50 out of 680 women (6.20\%) and this test detected nine 
cases with precancerous lesions, which included five CIN 1, three CIN 2 and one case of CIN 3. Downstaging missed one case of CIN 3 that was detected by VIA and VILI. Among 50 cases, 34 were cervicitis and seven were normal, which lead to its high false-positive rate (6.58\%). The sensitivity of downstaging in the present study was less (54.55\%) as compared to the study conducted at New Delhi (81.7\%), but the specificity of both studies was almost similar. Higher detection rate in other study was perhaps same because two ANMs were being involved with this work throughout the study period. ${ }^{6}$ There was another study where downstaging missed 15 out of 25 cases of dysplasia and carcinoma in situ. The precancerous lesions were missed because paramedical workers were less experienced. Authors concluded that this test could not be used as primary screening. A study conducted at Kerala showed sensitivity and specificity of $92.6 \%$ and $37.7 \%$ respectively. High sensitivity was observed in this study because it was performed on symptomatic population. ${ }^{7}$ Thus, this test could not be used as an independent screening modality but it could be used in areas with non-availability of acetic acid and Lugol's iodine. In the present study, the sensitivity of downstaging was less as compared to other studies, because it was not conducted on high risk and symptomatic patients.

In the present study, 40 out of 680 women showed positive VIA results. VIA detected seven precancerous lesions, out of which three were CIN 1, two were CIN 2 and two were CIN 3. VIA missed two cases of CIN 1 and one case of CIN 2 that were detected by VILI.

Various studies have shown sensitivity of VIA ranging from $55-97 \%$ and specificity ranging from 33-93\%. ${ }^{8-11}$ In the present study, the sensitivity of VIA was $63.64 \%$ which was less in comparison to other studies. White coherent light source is required for accurate interpretation of VIA results. The lack of this facility in rural area was a contributory factor for poor sensitivity of VIA in our study.

In a study, which was conducted at Egypt, the sensitivity was $97 \%$, this was because the local ethics committee did not approve any further testing of women with negative screening results without a sound indication. This restriction created a verification bias and limited statistical analysis. The sensitivity of VIA diagnosing CIN was $97 \%$, which was in agreement with the findings of most published trials. ${ }^{12}$

The sensitivity of VIA in a study conducted at Zimbabwe in the primary health center was $76.7 \%$, which was low as compared to other studies but higher than the present study. Low test quality was observed for VIA because of poor service delivery conditions like poor lighting, examination table and speculae. $^{13}$

In the present study, the specificilty of VIA was $93.42 \%$, which was comparatively higher than other studies. The specificity was $30.4 \%$ in a study conducted at Lahore, whereas it was $64.17 \%$ in the study conducted at Zimbabwe. ${ }^{14}$

The positive predictive value of VIA was poor in the present study. Similar results were noted in a study conducted by Sankaranarayanan R et al (17\%). ${ }^{15}$
In the present study, 27 cases were VILI positive, out of which 10 cases were detected as CIN, which included five cases of CIN 1, three cases of CIN 2 and two cases of CIN 3. VILI missed one case of CIN 1, which was detected by VIA whereas VILI detected three cases of CIN 1 and one case of CIN 2, which was missed by VIA.

In various studies, the sensitivity and specificity of VILI ranges from 78 to $92 \%$ and 74 to $85 \%$ respectively. ${ }^{6,11,14}$ In the present study, the sensitivity was same (90\%) as in the study conducted by Sankaranarayanan et al (91.7\%), whereas the specificity was comparatively higher $(97.46 \%) .{ }^{14}$

The diagnostic efficacy of VILI was found to be more as compared to VIA in the present study. These findings were similar to the study done by Sankaranarayanan et al. ${ }^{15}$ This could be attributed because the yellow color changes associated with a positive VILI test result could be recognized with much greater ease by trained health workers as compared to the acetowhite lesions associated with VIA.

The results of the present study indicate that VIA and VILI can be used as screening modalities in low resource settings because they are simple and inexpensive. Downstaging is not a suitable primary screening modality as it has poor sensitivity. However, it can be used in the rural areas where acetic acid and Lugol's iodine are not available. The negative predictive values of the tests are better than the positive predictive values.

\section{ACKNOWLEDGMENTS}

We acknowledge the help and support rendered by Dr Soudagar, Medical Officer, Kinaye PHC and the women who participated in the study.

\section{REFERENCES}

1. Mahmoobeh S, Diane S, Philip EC. Cervical cancer prevention: Cervical screening, science in evolution. Obstet Gynecol Clin N Am 2007;34:739-60.

2. Sehgal Ashok. Human papilloma virus (HPV) and screening strategies for cervical cancer. Indian journal of Med Res 2009;130:234-40.

3. Nanda Kavita, Douglas C McCrory, Evan R Myers. Accuracy of the Papanicolaou test in screening for and follow-up of cervical cytologic abnormalities: A systematic review. Ann Intern Med 2000;132:810-19.

4. Saloney N. Unaided visual inspection of the cervix: Clinical downstaging picture atlas. Geneva WHO Collaborating Centre in Human.

5. IARC screening group. Chapter I: Anatomical and pathological basis of visual inspection with acetic acid and with Lugol's iodine.

6. Lugoma Ghislain Sangwa, Mahmud Salaheddin, et al. Visual inspection as a cervical cancer screening method in a primary health care setting in Africa. International Journal of Cancer 2006;119:1389-95.

7. Wright TC, Denny L, Kuhn L, Goldie S. Use of visual screening methods for cervical cancer screening. Obstet Gynecol Clin N Am 2002;29:701-34.

8. Abdel-Hady ES, Emam M, Al-Gohary A, Hassan M, Farag MK, Abo-Elkheir M. Screening of cervical carcinoma using visual inspection with acetic acid. International Journal of Gynaecology and Obstetrics 2006;93(2):118-22. 
9. Belinson Jl, Pretorius RG, Zhang WH, Wu LY, Elson P. Cervical cancer screening by simple visual inspection after acetic acid. Obstet Gynaecol Sep 2001;98(3):441-44.

10. Khan S, Jha R, et al. Accuracy of cytology, visual inspection with acetic acid or Lugol's iodine in cervical cancer screening in Nepal. Journal of Obstet and Gynae 2007;292:48-53.

11. Basu PS, Sankaranarayan R. Visual inspection with acetic acid and cytology in the early detection of cervical neoplasia in Kolkata, India. International Journal of Cancer 2003;13(5):626-32.

12. University of Zimbabwe/JHPIEGO Cervical Cancer Project. Visual inspection with acetic acid for cervical screening: Test qualities in a primary-care setting. Lancet 1999;353:869-73.
13. Saira AS, Batra S, Gandhi G, Zutshi V. Comparison of methods used for screening of cervical preinvasive lesions. Journal of Obstet Gynecol India 2003;3:70-71.

14. Sankaranarayanan R, Parthasarathy Basu, et al. Accuracy of visual screening for cervical neoplasia: Results from an IARC multicentre study in India and Africa. International Journal of Cancer 2004;110(6):907-13.

15. Sankar Narayanan R, Wesley R, et al. Visual inspection of the uterine cervix after application of acetic acid in the detection of cervical carcinoma and its precursors. International agency for research of cancer 1998. 